y aura à la Havane une assemblée suprême et un comité exécutif.

La première sera composée d'un président effectif, qui sera en même temps le délégué officiel, nommé par le gouvernement, de deux vice-présidents, d'un secrétaire-général, d'un vice-secrétaire, d'un trésorier, d'un vice-trésorier, d'un comptable, d'un vice-comptable, d'un directeur en chef, de huit membres et d'un inspecteur général.

Le comité exécutif se compose du président, des vice-présidents, du secrétaire-général, du trésorier, du comptable et du directeur en chef.

Le comité exécutif répartit lui-même les charges entre ses membres.

Le règlement général organique déterminera les devoirs et les droits correspondant à chacune de ces charges. Le droit de faire des paiements appartient au président de l'assemblée suprême et du comité exécutif, lequel pourra déléguer ce pouvoir au premier vice-président.

Le décret gouvernemental a été publié dans la Gazette ufficielle du 14 octobre 1911.

\title{
Relations officielles entre la Groix-Rouge et l'Armée
}

Ces rapports sont définis par le décret n 85 du 5 février 1910, sur les relations de la Croix-Rouge avec le corps de santé militaire de l'armée cubaine. En voici la teneur :

Article Premier. - La Société nationale cubaine de la CroixRouge, établie dans la République de Cuba sous les auspices des conventions internationales de Genève de 1864 et de 1906, est un auxiliaire du corps sanitaire militaire et de la flotte, en temps de guerre, conformément à ce qui est prévu dans son règlement général organique, approuvé par le décret no 406 du 10 mai 1909 et par le Comité international de la Croix-Rouge le 7 septembre de même année; elle est, par suite, un corps volontảire de la réserve des forces ármées pour tout ce qui touche au but humanitaire qu'elle poursuit.

Art. 2. - Parmi les principaux éléments de secours que la CroixRouge pourra prêter à l'armée en campagne, il y a lieu de citer :

lo La création d'hôpitaux auxiliaires ou complémentaires de ceux établis par le corps de santé militaire,

$2^{\circ}$ Le transport de malades ou de blessés par trains-hôpitaux, par ambulances hippomobiles ou automobiles ou tirées par quelque moyen que ce soit, approprié aux nécessités ou aux circonstances, chaque fois que le corps de santé militaire lui fèra connaitre qu'il ne dispose pas des éléments nécessaires à ces services. 
$3^{0}$ L'établissement de postes de secours ou d'ambulances à postes fixes.

ART. 3. - Tout auxiliaire volontaire, en provenance de n'importe quelle corporation, institution ou association, doit être présenté et associé aux forces armées par l'intermédiaire de la seule Société nationale cubaine de la Croix-Rouge.

ART. 4. - Le président représentera la Croix-Rouge cubaine dans ses rapports avec le Secrétaire du gouvernement; les autorités militaires pourront inspecter le service des chefs d'ambulances, d'hôpitaux, etc., installés par la Croix-Rouge et le corps sanitaire militaire pourra veiller à ce que le combattant soit secouru de la meilleure manière.

ART. 5. - Le délégué du secrétariat du gouvernement auprès du Comité de la Croix-Rouge cubaine sera un membre du corps de santé militaire.

ART. 6. - Auprès de chaquue caserne, ou quartier général, il y aura un délégué de la Croix-Rouge cubaine représentant cette Société.

ART. 7. - Ce délégué, après autorisation du général en chef des forces armées ou du général en chef des troupes en campagne, s'entendra avec le chef du corps sanitaire dépendant de ce dernier, pour l'établissement d'hôpitaux et de postes de secours.

Art. 8. - Les autorités militaires, en leur qualité d'inspecteurs naturels de toutes les sections de la Croix-Rouge, établies sur leur terrain, veilleront au bon fonctionnement de l'institution, soit ellesmêmes, soit en déléguant à cette inspection un chef quelconque des forces armées et du corps de santé militaire.

ArT. 9. - Tout service ou installation de la Croix-Rouge cubaine devra porter son emblème.

Art. 10. - Le personnel de la Croix-Rouge cubaine, une fois accepté par l'autorité militaire, est soumis aux lois et règlements militaires. En temps de guerre ou en manceuvres, si les services du personnel ou du matériel de la Société deviennent nécessaires, l'autorité militaire en fait la demande au président de l'Association.

Art. 11. - Pour porter secours en qualité de médecin pratiquant, pour faire une opération aux soldats appartenant aux forces armées, il est nécessaire de posséder le diplôme de docteur ou de licencié en médecine, délivré par l'uniyersité nationale ou par une université étrangère, à la condition que ce dernier ait été rendu valable légalement.

ArT. 12. - La Croix-Rouge cubaine est responsable du personnel qui figure sur les listes qu'elle soumet aux autorités militaires.

ART. 13. - Elle veillera sous son entière responsabilité, à ce que les éléments sanitaires, qu'elle s'est engagée à foưnir à des endroits 
déterminés, ne manquent jamais à ses délégués ou représentants, à moins de cas dé force majeure, qui devront être immédiatement signalés au général en chef. A cet effet, toute installation nouvelle devra faire l'objet d'un rapport détaillé, mentionnant les ressources dont elle dispose et signalant à l'autorité militaire supérieure les éléments qui lui manquent, afin qu'en aucun cas, les malades ou les blessés qui y seraient envoyés ne manquent des ressources sur lesquelles croyaient pouvoir compter les autorités militaires ou le chef du corps de santé de l'armée. Le rapport devra faire mention du personnel, du matériel de logement et de transport, aussi bien que des médicaments, des instruments de chirurgie, des bandages et des vivres de chaque installation. Les augmentations ou diminutions seront signalées tous les dix jours, ou plus souvent si possible. La Société sera responsable du manque de services offerts par elle et acceptés par les forces armées.

ART. 14. - Les généraux des forces armées et les chefs commandant des colonnes établiront des certificats relatifs aux mérites du personnel de la Croix-Rouge; de même, le Comité exécutif de l'assemblée suprême de cette Société octroiera aux membres de l'armée, selon la hiérarchie, les distinctions honorifiques qu'elle possède, lorsqu'ils s'en seront rendus dignes.

ART. 15. - Les chefs de colonnes pourront accorder des rations de vivres et de fourrage au personnel et au bétail affecté au service de la Croix-Rouge cubaine, à la charge de l'institution quand son état de prospérité le permettra.

ART. 16. - Les autorités militaires résoudront toutes les questions relatives à l'installation d'infirmeries, de postes sanitaires ou d'hôpitaux pour l'assistance aux blessés ou malades à la charge de la Société et toujours derrière les lignes de combattants.

ArT. 17. - L'action de la Société ne pourra être étendue au service d'avant-garde ni aux hôpitaux d'évacuation, à moins de circonstances exceptionnelles qu'appréciera le général en chef ou celui à qui incombe la direction du combat.

ART. 18. - Pour maintenir les établissements précités dans la zone susmentionnée, leur transfert d'un lieu à un autre devra se faire conformément aux mouvements des troupes.

Art. 19. - Dans des circonstances extraordinaires, on pourra utiliser les services de la Croix-Rouge cubaine, surtout lorsqu'il s'agit d'événements prolongés, ou de marches rapides, qui forcent les chefs à séparer le gros des troupes des hôpitaux ou des ambulances ; la Croix-Rouge remplacera alors les ambulances et on lui confiera les malades qui ne peuvent suivre leur corps.

Art. 20. - Pour ce qui concerne la manière d'effectuer les changements de résidence, les haltes à faire et les endroits désignés 
pour le repos du personnel et du matériel de la Croix-Rouge cubaine, ainsi que pour ce qui a trait à la présence de celle-ci sur le champ de bataille, après le combat, pour seconder le corps de santé militaire, on appliquera les instructions du général en chef de l'armée en campagne et les prescriptions du règlement général organique déjà eité.

ART. 21. - La Croix-Rouge cubaine fournira un matériel de logement, de transport et un matériel chirurgical identique à celui de l'armée ; s'il en différait, il devrait, avant tout usage, être déclaré utilisable par les chefs du corps sanitaire des divers quartiers généraux de l'armée.

Art. 22. - Les dépôts de matériel sanitaire pour les besoins de la Croix-Rouge cubaine sur le territoire où se déroulent les opérations de guerre seront autorisés, moyennant une demande préalable de la Société.

Art. 23. - La Société adressera chaque semestre, en temps de paix, aux chefs des quartiers généraux, un mémoire détaillé des res. sources dont elle dispose en personnel et en matériel.

Art. 24. - Au moment d'entrer en campagne, quel que soit le temps écoulé depuis le moment où elle a fourni le précédent mémoire, elle adressera un rapport aussi détaillé que possible sur sa situation au double point de vue, personnel et matériel.

ArT. 25. - Avec l'autorisation du général en chef ou de celui qui dirige le combat, les membres de la Croix-Rouge cubaine pourront identifier les morts.

ART. 26. - Avant de répartir des dons ou des secours aux blessés et aux malades de l'arméc, le délégué de la Croix-Ronge eubaine . sollicitera l'autorisation de l'autorité militaire ; sans cette permission, il ne pourra commencer la distribution des dons ou secours.

ART. 27. - Aucun membre de la Société nationale cubaine de la Croix-Rouge ne pourra prendre l'initiative d'accorder un congé, une permission à des soldats blessés ou malades ou de réformer ces derniers; l'intervention des sociétaires est limitée aux soins médicaux à donner à ces soldats.

Art. 28. - En cas de désertion d'un soldat recueilli dans un hôpital ou une ambulance, le médecin directeur en avisera immédiatement l'autorité militaire la plus rapprochée.

\section{Hôpitaux auxiliaires}

ART. 29. - On n'autorisera pas l'ouverture d'un hôpital de la Croix-Rouge cubaine sans s'être assuré qu'il possède tous les éléments nécessaires à l'assistance, au traitement et à l'alimentation des blessés ou malades militaires.

ART. 30. - On veillera à ce que l'installation de ces hôpitaux 
se fasse dans des édifices réunissant les meilleurs conditions d'hygiène, dans des localités pourvues du plus grand nombre d'éléments d'assis. tance, sous tous leurs aspects, et desservies par les voies de communications les plus appropriées et les plus rapides par rapport à l'armée et au théâtre des opérations.

ArT. 31. - Les hôpitaux de campagne à la charge de la CroixRouge, aux conditions antérieurement indiquées, ne devront pas avoir, en moyenne, plus de 100 lits, répartis all nombre de 25 par salle de malades ou de blessés; le corps de santé militaire devra pouvoir ainsi veiller à l'assistance donnée à ces derniers avec les facilités que lui concèdent les articles 4 et 8 .

Art. 32. - Le personnel de la Société sera nommé suivant la forme réglementaire.

Arr. 33. - La Société veillera à ce qu'il y ait dans les hôpitaux au moins un médecin par salle ou section, avec le personnel subalterne nécessaire ; il devra y avoir également un médecin de garde pour faire face aux exigences du service.

ART. 34. - Les menus seront, à moins de force majeure, conformes à ceux des hôpitaux militaires. Les incidents qui pourraient surgir à ce propos et au sujet des prescriptions médicales seront consignés dans un rapport quotidien, signé par le chef de clinique et qui pourra être examiné par le corps de santé militaire.

Art. 35. - Le prix de séjour des individus hospitalisés dans ces étahlissements ne pourra excéder, en cas de paiemert par l'Etat, le prix fixé pour des séjours de ce genre dans les hôpitaux militaires.

ART. 36. - S'il surgissait une maladie contagieuse ou infectieuse, ou une épidémie, on devra en rundre compte immédiat ement à l'autorité militaire et au chef du corps de santé de l'armée.

ART. 37. - I.e rapport quotidien contenant le chiffre des décès, etc... sera également identique à celui des hôpitaux de l'armée; il devra faire prouve de la rilus grande exactitude dans la partie relative gux morts.

Arr. 38. - Dans des cas urgents, résultant des multiples incidents d'une campagne où les blessés ou malades entreraient à l'ambulance avec leur équipement et leurs armes, cet équipement serait placé dans des magasins convenables, de façon à ne pas se détériorer et à ne pas être volé ; il en sera donné une description détaillée à l'autorité militaire de la place ou du camp le plus rapproché, et au délégué de la Croix-Rouge cubaine auprès du général en chef, afin que celui-ci en ait connaissance. On agira de même pour les valeurs trouvées en la possession des malades ou blessés; ces valeurs entreront dans la caisse de l'établissement; on en donnera un reçu aux intéressés et on les leur rendra le jour de leur sortie. En cas de décès, les valeurs seront 
remises au corps auquel appartenait le soldat décédé. S'il existe dans la localité un parc d'artillerie ou s'il s'y trouve des troupes, les munitions et les armes du soldat mort seront immédiatement remises au chef de ces forces.

ArT. 39. - Outre qu'elle devra s'en tenir, ainsi que cela a déjà été dit, pour ses rapports journaliers, aux modèles en vigueur dans les hôpitaux militaires, la. Croix-Rouge cubaine devra posséder dans ses bureaux les règlements du service sanitaire en campagne, ceux des hôpitaux militaires et tous ceux concernant le service du corps de santé de l'armée, pour pouvoir approprier ses règlements à ceux précités, dans la mesure du possible, et pour pouvoir trancher toutes les questions qui provoqueraient une hésitation de sa part.

\section{Service de la Croix-Rouge cubaine, sur les voies ferrées, fluviales et maritimes}

ArT. 40. - Le transport des blessés ou malades, derrière les lignes de troupes, peut être confié à la Croix-Rouge cubaine, quand le corps sanitaire militaire le juge opportun.

ArT. 41. - Le général en chef détermine la composition des équipes accompagnant les trains-hôpitaux et le reste des services incombant à la Croix-Rouge cubaine.

ArT. 42. - Ces trains ne porteront pas de signe spécial (à part ceux prévus par les règlements de chemins de fer), autre que l'emblème de la Croix-Rouge cubaine.

Art. 43. - Chaque fois que ce sera possible, un chef on un officier du corps de santé militaire, ou tout au moins un officier de l'armée, accompagnera ces trains ; il sera chargé de veiller au transport des blessés ou malades et de leur témoigner la sollicitude à laquelle ils ont droit.

ART. 44. - Lorsqu'on organisera des trains-hôpitaux à la charge de la Croix-Rouge cubaine, il y aura lieu d'établir un rapport complet et d'une exactitude scrupuleuse. Ce rapport contiendra une liste détaillée des soldats transportés et fera mention de tous les incidents survenus au cours du voyage. Il sera établi en triple expédition; l'un des exemplaires sera conservé par le médecin, chef du convoi, qui en remettra un second au médecin du corps de santé militaire de la localité de départ; celui-ci signera l'exemplaire qui reste entre les mains du médecin chef de convoi, qui, à son tour, signera la prise en charge des blessés ou malades sur l'exemplaire qu'il laisse au chef du corps sanitaire ou à l'autorité militaire, et à dófaut de ceux-ci, au maire ou à l'autorité civile de la localité où a lieu le départ.

ART. 45. - Le médecin chef de convoi refusera tout individu 
exposé par la gravité de son état ì mourir en cours de route, ou à être dangereux pour les autres. Si, dans des circonstances extraordinaires, il était obligé de l'admettre, il ferait constater le cas dans le rapport (journal de train) pour sauver sa responsabilité professionnelle.

ART. 46. - La Croix-Rouge nationale cubaine pourra établir des postes de secours, convenablement dotés, dans les gares de chemins de fer situées près du théâtre des opérations, dans les ports de débarquement habituel des blessés ou malades arrivés par les voies maritimes, fluviales ou ferrées et, en général, dans les localités où le passage des troupes en temps de guerre est probable. Ces postes de secours devront être pourvus des éléments sanitaires nécessaires. Ils devront en outre :

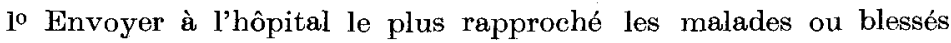
qui ne peuvent continuer le voyage.

20 Secourir ceux qui sont gravement atteints et les conduire ensuite à l'hôpital.

$3^{\circ}$ Donner des médicaments et des soins à ceux qui souffrent de blessures légères pour leur permettre de poursuivre leur route.

$4^{\circ}$ Provoquer la répartition des malades ou blessés entre les hôpitaux locaux et faciliter leur transport à ces hôpitaux.

$5^{\circ}$ Porter secours immédiatement au blessé qui le réclame.

Le matériel affecté à ces postes de secours sera celui fixé par le règlement et sera soumis à l'inspection de l'autorité militaire et au chef du corps de santé de l'armée. Il devra y avoir dans chaque poste de secours un médecin aidé du personnel subalterne ad hoc.

ART. 47. - La Croix-Rouge pourra être autorisée à utiliser, pour le transport fluvial et côtier des blessés et malades, des bateauxhôpitaux soumis aux mêmes règlements que ceux édictés pour les trains. Dans le second cas (c'est-à-dire pour ce qui concerne les transports le long des côtes), il y aura lieu de faire état des dispositions prises par la marine nationale.

Services de la Croix-Rouge dans les villes en état de siège, pendant les émeutes, etc.

ART. 48. Quand l'ordre public est troublé, en temps de paix, la Société pourra installer des postes de secours ou des hôpitaux et porter secours aux blessés, sans s'interposer entre les combattants.

ArT. 49. - Ces postes de secours et hôpitaux seront indiqués à l'autorité militaire qui saura ainsi où ils se trouvent et pourra les approuver ; elle devra en avoir connaissance, soit que les postes aient été installés depuis que l'autorité militaire a pris le commandement de la ville, soit qu'ils aient existé antérieurement.

Art. 50. - Dans les villes en état de siège, la Société, quand elle portera secours à la troupe, se conformera au présent règlement 
sur les postes de secours et hôpitaux. Elle pourra, dans ces établissements, seconder le service de santé militaire, chaque fois que cela lui paraîtra nécessaire.

\section{Dispositions générales}

ART. 51. - Les postes de secours, hôpitaux et autres services de la Croix-Rouge cesseront de fonctionner quand le corps de santé militaire lui aura fait connaître qu'il dispose de tout le matériel et personnel nécessaires pour l'assistance aux blessés et malades militaires.

ART. 52. - La Société ne pourra, en aucun cas, retirer son personnel ou son matériel des endroits où elle a été autorisée à les mettre par le chef des forces armées, sans le consentement de ce dernier.

ART. 53. - Les prisonniers faits à l'ennemi seront l'objet des mêmes soins que les nationaux, mais toujours avec l'autorisation de l'autorité militaire, qui prendra les mesures utiles pour leur garde et leur sécurité.

ART. 54. - Les sociétaires de la Croix-Rouge pourront recevoir à leur domicile particulier, des blessés ou malades, moyennant que l'autorité militaire les y autorise et qu'il s'agisse de convalescents ou de soldats grièvement blessés ou assez malades pour être empêchés pendant longtemps de retourner sur le théâtre des opérations.

ART. 55. - Le présent règlement entrera en vigueur à la date de sa publication dans le Journal officiel et aura son effet à partir du moment où la Société nationale cubaine de la Croix-Rouge entrera en fonctions conjointement avec les forces armées de la nation.

ART. 56. - Afin qu'il règne toujours la plus parfaite harmonie entre les membres de la Croix-Rouge cubaine et ceux des forces armées, et la plus complète discipline, il est établi qu'ils respecteront mutuellement leur hiérarchie.

Le secrétaire du Gouvernement est chargé de l'exécution du présent décret qui entrera en application à la date de sa publication dans le Journal offlciel ${ }^{1}$.

\section{Le Secrétaire,}

F. LOPEZ-LEIVA
Le président,

José Gomez

${ }^{1}$ Ce décret a été publié dans la Gazeta oficial du 7 février 1910. 